\title{
Céramiques ottomanes de tradition byzantine d'Iznik
}

Véronique François

Citer ce document / Cite this document :

François Véronique. Céramiques ottomanes de tradition byzantine d'Iznik. In: Anatolia Antiqua, Tome 4, 1996. pp. 231-245;

doi : 10.3406/anata.1996.1200

http://www.persee.fr/doc/anata_1018-1946_1996_num_4_1_1200

Document généré le 14/05/2016 


\section{CERAMIQUES OTTOMANES DE TRADITION BYZANTINE D'IZNIK}

A l'appellation "céramique ottomane d'Iznik", nous avons coutume d'associer les plus fameuses d'entre elles, c'est-à-dire les céramiques dites de Milet et les faiences peintes polychromes, qui ont fait la renom-

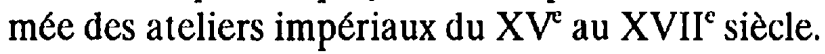

Mais parmi les productions d'époque ottomane, on distingue toute une série de vases à glaçure plombifère, de facture assez grossière et de techniques diverses. Rarement prise en compte dans les publications, cette céramique doit constituer, pourtant, une grande part de la production ottomane la plus courante. En effet, il est vraisemblable que l'installation des Ottomans n'ait pas radicalement modifié les habitudes culinaires et les usages de la table. On a continué à manger dans de la vaisselle à glaçure, qui semble d'ailleurs avoir envahi la cuisine, comme on peut le remarquer dans les fouilles de Saraçhane, où on observe une augmentation notable des tessons à glaçure au dépend de la céramique commune à paroi fine, non glaçurée ${ }^{1}$. De la même façon, il serait absurde d'envisager une rupture radicale entre les pratiques des artisans byzantins -détenteurs d'un savoir-faire éprouvé-et celles des potiers ottomans, leurs descendants directs. Et c'est sans doute sous l'aspect de la continuité, et non pas de la rupturc, que doivent être envisagées certaines de ces productions ottomanes.

Paradoxalement, cette céramique est souvent ignoree du fait, justement, des grandes analogies qu'elle entretient avec les productions byzantines tar- dives, dont elle ne se distingue pas toujours aisément. En effet, ces vases sont à considérer comme les héritiers directs des traditions byzantines : la pâte est argileuse ; les décors sont incisés ou champlevés à travers une couche d'engobe, parfois rehaussés de pigments colorés, ou peints à l'engobe ; les glaçures sont plombifères et colorées dans les mêmes tonalités que les céramiques byzantines; seules les formes et les tailles se distinguent.

Sur les sites ${ }^{2}$, confrontés à des fragments de ce genre il n'est pas toujours facile de les attribuer à l'époque ottomane, aussi il nous a semblé intéressant de publier ici un certain nombre de vases produits à Iznik -comme en témoignent les nombreux vases rejetés en cours d'élaboration (fig.1) - afin d'offrir au chercheur un panorama plus élargi des productions ottomanes en même temps qu'un lot de référence.

Ce matériel, entreposé au Musée, a été retrouvé à l'occasion de divers travaux de creusement effectués dans la ville, notamment dans une tranchée percée à l'arrière des bâtiments de la Poste, Kulıçarslan Caddesi, en $1987^{3}$. Il est complété par la collection de vases présentée dans les vitrines du Musée - ces vases provenant des fouilles menées dans le centre d'Iznik, fouilles des ateliers ottomans ${ }^{4}$ et du thếtre romain ${ }^{5}$.

1) J. W. Hayes, Excavations at Saraçhane in Istanbul, vol. 2 : The Pottery, Princeton, 1992, p. 233, 327-333.

2) Pour des découvertes en contexte à Istanbul voir: D. 'Talbot-Rice, "Excavations at Bodrum Camii 1930", Byzantion 8, 1933, p. 173-174; J. W. Hayes, "The Excavated Pottery from the Bodrum Camii", in C. L. Striker, The Myrelaion (Bodrum Camii) in Istanbul, Princeton, 1981, p. 36-41.

3) Outre ces fragments, la céramique ollomane était largement représentée par des exemplaires de Miletus Warc.

4) O. Aslanapa, S. Yctkin, A. Altun, The Iznik Tile Kiln Excavations (The Second Round: 1981-1988), Istanbul, 1989.

5) On attend avec beaucoup d'impatience la publication des céramiques byzantines et ottomanes, retrouvées en très grande quantité au théátre, pour avoir une idée complète des productions d'lznik. B. Yalman, "Iznik Theatre, 1982", AnatStud, 33, 1983, p. 250-252; ibid, "Iznik Thcatrc, 1983", AnatStud, 34, 1984, p. 222-223 ;ibid, "Iznik 'Tiyatro Kazısı 1991", XIV. Kazı Sonuçları Toplantısı, II, Ankara 25-29 mayıs 1992, Ankara, 1993, p. 181-203. 


\section{LA TRADITION BYZANTINE A IZNIK ET LES CARACTERISTIQUES COMMUNES AUX DEUX PRODUCTIONS}

Du $X^{\mathrm{c}}$ au XIII ${ }^{\mathrm{c}}$ siècle, Nicée apparaît comme un centre de production de céramiques byzantines à pâte blanche et à pâte rouge ${ }^{6}$. Ces dernic̀res sont, pour la plupart, des vases de petite taille avec des parois tres fines. Ce sont majoritairement des formes ouvertes, petites coupes montées sur unc base annulaire caractéristique. Les décors sont traités au sgraffito, incisés, champlevés à travers une couche d'engobc, ou peints à l'engobe. Parfois, ils sont rchaussés de pigments colorés verts ou bruns. Les glaçures plombifères sont très brillantes, surtout de couleur caramel, mais elles peuvent être aussi vert pâle ou vert bouteille, jaune pâle ou jaune d'or. L'utilisation de la pernette est attestée par les fréquentes traces d'arrachement. La pâte argileuse, bien épurée - suffisamment pour permettre dc tourner des vases avec des parois de 2,3 , ou $4 \mathrm{~mm}$ d'epaisseur-, est fine, de coulcur orange, parfois rouge brique. Bien cuite, très dure, elle a unc sonorité clairc.

Les quclques vases ottomans présentés ici partagent un certain nombre de caractéristiques avec ces tessons byzantins. La pâte d'abord cst identique et on n'en sera pas surpris. S'il existe à proximité de Nicéc des gisements d'argile exploités à l'époque byzantine, il y a peu de raison d'en abandonner l'extraction aussitôt après la conquête de la région par les Ottomans. Toutefois, si la pâte est la même, son traitement semble parfois moins soigne. Il arrive qu'elle soit grossièrement travaillé ; les parois vues en coupe sont moins homogènes. Une couche d'engobe est appliquée sur la plupart des fragments. Les vases incisés, rejetés avant l'application de la glaçure, montrent bien une couche d'engobe épaisse, rosée ou beige. Les techniques de traitement de surface sont les mêmes que celles utilisées pour la céramique byzantine : l'incision moins soignée, tracée avec probablement plus de rapidité, creee des décors moins sophistiqués, parfois rehaussés de pigments colorés; les pigments utilisés pour les motifs peints sont le vert et le brun. Les glaçures sont encore des glaçures plombifères, également très brillantes, de tonalités assez foncées, plutôt du vert soutenu, du brun ou du caramel que du vert pâle ou du jaune pâle.

\section{I.ES PAR'TICULARITES OTTOMANES : FORMES ET 'TAILLES}

Forme $\Lambda$ (fig.2)

C'est la forme la plus fréquente: unc coupe à panse ronde, courbe continue et douce; les lèvres sont dans le prolongement de la panse, légèrement saillantes à l'extrémité ; la base annulaire a un profil arrondi. Le diamètre supéricur est compris entre 164 et $230 \mathrm{~mm}$, pour une hauteur comprise entre $83 \mathrm{ct} 92 \mathrm{~mm}$. Cette formc caractérise surtout les vases à glaçure monochrome (4-10,11), mais pas uniquement, puisque un vase peint polychrome (12) est de même type. Ce profil n'est pas sans rappeler celui de ccrtains exemplaires de Miletus Ware - produits à Iznik, de la fin du XV sic̀cle au début du XVI courbc $^{7}$ mais aussi par les dimensions. ${ }^{8}$. Des vases au profil identique, retrouvés dans les fouilles de Bodrum Camii et Saraçhane Camii à Istanbul, sont datés du XVI" siècle.

Sans présager de la fonction du vase, on peut remarquer que, parmi les productions ottomanes traditionnelles du début du XVI ${ }^{\mathrm{e}}$ siècle, cette forme cst généralement caractéristique des kâse ${ }^{9}$, coupes à sorbet ou à compote, utilisécs en tous les cas pour contenir des substances à forte teneur en liquide, ce qui justificrait leur profondeur (diamètre compris entre $196 \mathrm{et} 216 \mathrm{~mm}$ pour unc hauteur entre $84 \mathrm{ct} 112 \mathrm{~mm}$ ).

\section{Forme B (fig.3)}

Grande coupe avec panse à courbe discontinue marquée par un point d'intersection ${ }^{10}$ sur le bas de la panse ct un point d'inflexion ${ }^{11}$ sur le haut de la panse ; la lèvre, à extrémité arrondic, se trouve dans le prolongement de la panse ; la base est annulaire et le profil du pied variable $(2,3,14,15,30,31)$. Ce sont de grands vases (diamètre supérieur compris entre 180 et 240 $\mathrm{mm}$ pour une hauteur entre $90 \mathrm{ct} 115 \mathrm{~mm}$ ), aux formes lourdes et aux parois épaisses.

\footnotetext{
6) Etude en cours menée par l'auteur.

7) Voir par exemple : J. W. Haycs, 1992, p. 240, fig. 92, 11, 13, 19, 20.

8) Les exemplaires de Saraçhanc ont un diamètrc compris entre $170 \mathrm{ct} 280 \mathrm{~mm}$.

9) N. Atasoy, J. Raby, Jznik. La poteric en Turquie ottomane, Paris, 1990, p. 38, fig. 25, p. 45.

10) Point d'intersection : point de rupture d'une courbe provoquant un changement brusque de contour, formant un angle saillant ou ren-

11) Point d'inflexion : point d'inversion sans rupture entre segments convexes et concaves d'une courbe continue.
} trant. 


\section{Forme C (fig. 2)}

Elle ne s'illustre que par un seul vase à fond plat (1) avec une panse marquée par un point d'intersection et une lèvre très saillante soulignée à l'intéricur par une arête.

Les formes A et B sont surtout caractéristiques des céramiques à glaçurc monochrome. Les vases incisćs, représentés notamment par une série de bords, montrent une plus grande diversité dans les profils (fig. 4) : panse quasi rectiligne avec levres dans le prolongement $(21,32)$; lcvres très saillantes par rapport à la panse allant même jusqu'au marli (22-25).

\section{TECIINIQUES ET DECORS}

\section{La céramique à glaçure monochrome}

Les glaçures - toujours appliquecs sur une couche d'engobe- brunes, caramel, vertes ou jaune d'or sont très brillantes. A l'extérieur du vase, le haut du bord est généralement couvert d'engobe et de glaçure, parfois l'engobe est appliqué jusqu'en bas de la panse (7, fig. 2), ou bien il arrive également que la pâte soit laissée nue. L'emploi de pernette est attesté par les traces d'arrachement, une pernette est même restéc collée au fond du vase 11 (fig. 1,2). Cette sćrie partage les formes du Miletus Ware. Particulièrement difficile à dater ce type de céramique, au regard des découvertes de Saraçhane (type Ware $C$ ), daterait de la première moitié du $\mathrm{XVI}^{\mathrm{e}}$ siccle $^{12}$.

\section{La céramique peinte}

Elle est représentée par deux fragments de type distinct. Le premier est assez habituel à Iznik : on rctrouve des exemples proches dans les fouilles des fours ottomans de la ville ${ }^{13}$. Ils sont attribués aux $X I V^{e}-X^{e}$ siecles. Sur une couche d'engobe des pigments colorés verts et marron sont appliqués sous la forme de coulures plus ou moins maîtrisées, ici en chevrons imbriqués $(12$, fig. 2,5$)$. Le second type est tout à fait caractéristique d'une production non encore identifiée retrouvée à Saraçhane ${ }^{14}$ et à Thasos en Egée du Nord (fig. 5). Un motif central - cercles concentriques, spirale, palmette stylisee, fleur stylisée- est peint sur engobc cn vert, ocre ou marron. La glaçure est jaune pâle.
Les traces laissées par le trépied témoignent bien de son utilisation. A Thasos et à $\mathrm{I} \%$ nik, ces vases ont été trouvés hors de tout contexte chronologique. A Istanbul, ce type est daté du second quart du XVI" siècle.

\section{La céramique incisée}

Pour les catégories présentées ici, des vases rejetés en cours d'élaboration et des ratés de cuisson attestent bien une production locale. Une premic̀re séric se distingue par son décor : un large médaillon central orne d'une fleur incisée retrouvé notamment sur plusieurs fonds engobés mais rejetés avant l'application de la glaçurc (26, fig. $8 ; \mathbf{2 7}$, fig. 5 ). Un vase entier nous permet de restituer le décor complet : sur la panse, une succession de spirales et sur le bord, des cercles concentriques incisés et une ligne onduléc (14, fig. 3, 5). Autres décors caractéristiques : un médaillon central orné de hachures - dans le style des blasons incisés sur les céramiques mamelouks-puis une série de quatre cercles concentriques et sur la panse, des spirales incisées (30, fig. 3,6;31, fig. 3,6); dans un large médaillon occupant tout le fond, délimité par deux cercles concentriques, une fleur est grossièrement tracéc (15, fig. 3,$6 ; 28,29$, fig. 2,6 ). Les fragments de bords sont souvent ornés de bandeaux de chevrons incisés rehaussés de pigments verts et bruns sur une glaçure jaune clair très brillante ou verte $(\mathbf{2 2}$, fig. 4,$7 ; \mathbf{2 3 - 2 5}$, fig. 4), ou encore d'une ligne ondulée prise entre deux cercles concentriques (21, 32, fig. 4, 7). Enfin, divers autres vases, de facture plus grossière sont ornés de fleurs très stylisées $(\mathbf{1 8 , 2 0}$, fig. $\mathbf{8})$ ou d'un gros noud de Salomon (19, fig. 8).

Pour ces vases aucune datation sûre ne peut être avancée, bien qu'il existe quelques similitudes avec du matériel daté du XVI ${ }^{\mathrm{c}}$ siècle découvert dans les fouilles de Bodrum Camii ${ }^{15}$.

Ces productions variees, bien que modestes, lorsqu'elles peuvent être datées, sont attribuces aux XIV ${ }^{c}$ $X V^{e}$ siècles et plus sûrement au second quart et à la première moitié du XVI ${ }^{\mathrm{e}}$ siècle. De par les analogies qu'elles entretiennent avec la céramique byzantine, elles paraissaient plutót représentatives d'une production transitoire. En fait, elles en sont assez éloignées chronologiquement et sont contemporaines des meilleures productions de faïence d'Iznik, avec lesquelles 
elles sont souvent associees sur les sites. Ce qui prouve, d'une part, que la tradition byzantine s'est maintenue bien au-delà de ce qu'on imaginait et, d'autre part, que cohabitaient à Iznik deux types d'ateliers. Dans les premiers, on fabriquait des faïences peintes monochromes et polychromes, vases de luxe destinés à la cour ; dans les seconds, on réalisait une vaisselle argileuse à glaçure plombifère qui répondait à une demande locale mais qui, probablement, était aussi commercialisée plus largement. Pour preuve de la diffusion de ce type de poterie, la céramique peinte de la seconde catégoric - dont il est vrai rien ne nous assure qu'elle ait été fabriquée sur place- retrouvée à Thasos, à Iznik et à Istanbul. Incontestablement issus d'une même production -dont il reste à découvrir l'origine - ces vases ottomans ont été objets de commerce, puisque distribués dans le Nord de l'Egée et dans la région constantinopolitaine. Par aillcurs, les vases d'Iznik entreticnnent de grandes ressemblances avec le matériel issu des fouilles de Constantinople. Dans la mesure où nous avons la preuve d'une production locale, nous sommes en droit de supposer l'existence d'un commerce avec la capitale, sans qu'il soit pour autant possible de préciser dans quelles conditions et par quels moyens cette commercialisation de'productions de vaisselle de table s'exerçait.

\section{CATALOGUE}

\section{Céramique à glaçure monochrome}

1 (SN 94) fig. 2

Forme : $\mathrm{O} / \mathrm{PC}^{16}$.

Pâte : argileusc, orange clair, fine.

Traitement de surface intérieur : engobe blanc; glaçure vert pré marquée de nombreux picots.

Traitement de surface extérieur : engobe et glaçure sur lc haut du bord et coulures de glaçure sur la panse. Sous le fond plat, traces très nettes du décollage du vase de la tournette. DSUP $^{17}=170 \quad \mathrm{DNL}=156 \quad \mathrm{HT}=55 \quad \mathrm{HNL}=46 \quad \mathrm{DSP}=74$ $\mathrm{DIP}=76 \mathrm{HEP}=6 \mathrm{EPF}=12$

\section{2 (IZ.95.53) fig. 3}

Forme : $\mathrm{O} / \mathrm{PC}$.

Pâte : argileuse, orange clair, mal travaillée.

Trt. de surf. int. : engobe rosé; glaçure vert kaki brillante.

Traces de pernette : un pied arraché et un arrachement de la pâte.

Trt. de surf. ext. : cngobe et glaçure sur le bord.

DSUP $=180 \mathrm{DNL}=180 \mathrm{DPI}=114 \mathrm{HT}=90 \quad \mathrm{HNL}=83$

$\mathrm{HPI}=24 \mathrm{DSP}=72 \mathrm{DIP}=76 \mathrm{HEP}=12 \mathrm{IIIP}=11 \mathrm{LPP}=6$

\section{3 (IZ.95.12) fig. 3}

Forme : $\mathrm{O} /$ bord.

Pâte : argileuse, brun clair avec de petites inclusions blanches. Trt. de surf. int. : engobe et glaçure caramel en coulures marquées par des picots noirs.

Trt. de surf. ext. : engobe et glaçure appliqués de façon irré- gulière sur le bord.

DSUP $=240 \mathrm{DPI}=232 \mathrm{IIT}=40 \mathrm{HPI}=28$

4 (IZ.95.13) fig. 2

Forme : $\mathrm{O} /$ bord et panse.

Pâte : argileuse, orange clair, finc, bien cuite.

Trt. de surf. int. : engobe rosé ct glaçure caramel très brillante, tressaillee.

Trt. de surf. ext. : engobe et glaçure sur le haut du bord.

$\mathrm{DSUP}=170 \mathrm{HT}=70$

5 (IZ.95.14) fig. 2

Forme : $\mathrm{O} /$ bord.

Pâte : argileuse, orange clair, fine, bien cuite.

Trt. de surf. int. : engobe et glaçure brune très brillante.

Trt. de surf. ext. : engobe et glaçure sur le sommet du bord 'et coulures sur la panse.

$\mathrm{DSUP}=220 \mathrm{HT}=30$

6 (IZ.95.15) fig. 2

Forme : $\mathrm{O} /$ bord.

Pâte : argilcusc, orange clair, très fine, bien cuite.

Trt. de surf. int. : engobe rosé et glaçure caramel.

Trt. de surf. ext. : engobe sur tout le fragment.

DSUP $=230 \mathrm{HT}=42$

7 (IZ.95.56) fig. 2

Forme : $\mathrm{O} /$ fond et panse.

Pâte : argileuse, brune, fine.

16) $\mathrm{O}$ (ouverte) ; $\mathrm{PC}$ (profil complet).

17) Toutes les dimensions sont données en millimètres. DSUP : diamètre supérieur ; DNL : diamètre à la naissance de la lèvre; DPI : diamètre au point d'inflexion; $H^{\prime} T$ : hauteur totale conservée; HNI : hauteur à la naissance de la lèvre ; HPI: hautcur au point d'inflexion ; EP' : epaisscur ; DSP : diamètre supéricur du pied ; DIP : diamètre inférieur du pied; HEP : hauteur extérieure du pied ; IIIP : hauteur intérieure du pied ; EPF : épaisseur du fond. 
Trt. de surf. int. : engobe rosé ; glaçure brune très brillante. Traces de pernette : trois pieds arrachés.

Trt. de surf. ext. : engobe appliqué au pinceau presque jusqu'en bas de la panse. Traces de glaçure çà et là.

$\mathrm{DSP}=69 \mathrm{DIP}=74 \mathrm{HEP}=9 \mathrm{HIP}=11 \mathrm{EPF}=8$

8 (IZ.95.57)

Forme : $\mathrm{O} /$ fond et panse.

Pâte : argileuse, orange clair, assez fine, bien cuite.

Trt. de surf. int. : engobe rosé ct glaçure caramel très brillante avec sortes de tavelures.

Traces de pernette : deux pieds arrachés.

Trt. de surf. ext. : aucun.

$\mathrm{DSP}=67 \mathrm{DIP}=74 \mathrm{HEP}=11 \mathrm{HIP}=8 \mathrm{EPF}=6$

9(IZ.95.58) fig. 2

Forme : $\mathrm{O} /$ fond et bas de la panse.

Pâte : argilcuse, orange clair, fine.

Trt. de surf. int. : engobe rosé et glaçure jaune d'or très brillante.

Traces de pernette : trois arrachements jusqu'à la pâte.

Trt. de surf. ext. : DSP $=68 \mathrm{DIP}=72 \mathrm{HEP}=14 \quad \mathrm{HIP}=12$ $\mathrm{EPF}=10$

\section{Vases rejetés en cours d'élaboration}

10 (IZ.95.54) fig. 2

Forme : $\mathrm{O} / \mathrm{PC}$.

Pâte : argileuse, orange vif, plutôt grossière avec inclusions noires.

Trt. de surf. int. : aucun. Vase tourné, cuit et rejeté avant l'application de la glaçure.

Trt. de surf. ext. : aucun.

DSUP $=180 \mathrm{DNL}=171 \mathrm{HT}=87 \mathrm{HNL}=82 \mathrm{DSP}=66$

$\mathrm{DIP}=68 \mathrm{HEP}=11$

11 (IZ.95.55) fig. 1, 2

Forme : O / PC.

Pâte : argilcusc, fine, brunâtre, surcuite.

Trt. de surf. int. : engobe rose ; glaçures jaune en coulures et vert foncé au fond, brillantes.

La pernette est restée collée au fond.

Trt. de surf. ext. : engobe et glaçure sur le bord et engobe jusqu'à mi-panse.

DSUP $=192 \mathrm{HT}=83 \mathrm{DSP}=70 \mathrm{DIP}=74 \mathrm{HEP}=9 \mathrm{HIP}=7$

$\mathrm{EPF}=10$

\section{Céramique peinte}

12 (IZ.95.59) fig. 2, 5

Forme : O / PC.

Pâte : argileusc, orangc, asscz finc malgré quelques inclusions blanches.

Trt. de surf. int. : engobe rose ; glaçure ocre très brillante et chevrons imbriqués peints en vert et marron.

Traces de pernette : un pied arraché et un arrachement de pâte.
Trt. de surf. ext. : engobe et glaçure sur le haut du bord. $\mathrm{DSUP}=164 \mathrm{HT}=92 \mathrm{DSP}=66 \mathrm{DIP}=70 \mathrm{HEP}=14 \mathrm{HIP}=18$ $\mathrm{EPF}=6$

\section{3 (IZ.95.60) fig. 2, 5}

Forme : $\mathrm{O} /$ fond.

Pâte : argileuse, orange clair, assez fine.

Trt. de surf. int. : engobe blanchâtre ; glaçure jaune clair, au centre une tache de pigments bruns et un cercle de pigments vert clair et en bas de la panse, un autre cercle de pigments bruns.

Traces de pernette : trois arrachements de la pâte. .

Trt. de surf. ext. : traces d'engobe.

$\mathrm{DSP}=66 \mathrm{DIP}=70 \mathrm{HEP}=15 \mathrm{HIP}=14 \mathrm{LPF}=8$

\section{Céramique incisée}

14 (IZ.95.67) fig. 3, 5

Forme : O / PC.

Pâte : argilcuse, ocre, fine, bien cuite.

Trt. de surf. int. : engobc beige ; sur le bord, deux cercles concentriques incisés puis une ligne ondulee et de nouveau deux cercles concentriques incises ; sur la panse, succession de spirales; au fond, dans un médaillon central délimité par deux cercles concentriques incisés, une fleur incisée. Glaçure vert kaki brillante.

Trace de pernette : un pied arrache.

Trt. de surf. ext. : engobe et glaçure sur le haut du bord, puis engobe seul jusqu'à mi-panse. Sous la base, un cône.

DSUP $=240 \mathrm{DNL}=230 \mathrm{HT}=115 \mathrm{HNL}=105 \quad \mathrm{DSP}=81$ $\mathrm{DIP}=84 \mathrm{HEP}=11 \mathrm{HIP}=5 \mathrm{RPF}=14$

\section{5 (IZ.95.62) fig. 3, 6}

Forme : $O /$ fond et bas de la panse.

Pâte : argileuse, orange clair, bien épuree mais grossièrement travaillee.

Trt. de surf. int. : engobe blanchâtre; dans un large médaillon occupant tout le fond, délimite par deux cercles concentriques, une fleur grossièrement incisée. Glaçure jaune clair, un cercle peint en brun.

Traces de pernette : trois arrachements de la pâte.

Trt. de surf. ext. : aucun.

$\mathrm{DSP}=80 \mathrm{DIP}=86 \mathrm{HEP}=10 \mathrm{HIP}=15 \mathrm{I}: \mathrm{PF}=10$

16 (P52) fig. 8

Forme : $\mathrm{O} /$ fond.

Pâte : argileuse, rouge brique, assez fine.

Trt. de surf. int. : engobe rosé ; un médaillon central, délimité par deux cercles concentriques incisés, occupe tout le fond, il est orné d'une fleur à cinq pétales. Glaçure brune, brillante. Traces de pernette : trois arrachements de glaçure.

Trt. de surf. ext. : engobe et glaçurc sur la panse et glaçure jusqu'au bas de la panse.

$\mathrm{DSP}=82 \mathrm{DIP}=86 \mathrm{HEP}=13 \mathrm{HIP}=17 \mathrm{EPF}=15$

17 (IZ.95.70) fig. 8

Forme : $\mathrm{O} /$ fond et bas de la panse. 
Pâte : argilcusc, orange vif, fine.

Trt. de surf. int. : cngobe beige ; médaillon central délimité par deux cercles concentriques, avec chevrons incisés, inscrit dans un hexagone aux côtés incurvés, dans chaque angle, une spiralc; sur la panse chevrons incisés. Glaçure vert sapin.

Trt. de surf. ext. : aucun.

$\mathrm{DSP}=77 \mathrm{DIP}=82 \mathrm{HEP}=11 \mathrm{HIP}=10 \mathrm{EPF}=11$

18 (IL.95.64) fig. 8

Forme : $\mathrm{O} /$ fond et bas de la panse.

Pâte : argilcuse, rouge vif, assez épurée mais travaillée grossièrement.

Trt. de surf. int. : au fond, une grosse fleur stylssée, incisée directement sur la pâte (aucune trace d'engobe). Glaçurc brune, brillante.

Traces de pernette : un pied arraché et deux arrachements de la glaçurc.

Trt. de surf. ext. : aucun.

$\mathrm{DSP}=83 \mathrm{DIP}=88 \mathrm{HEP}=12 \mathrm{HIP}=10 \mathrm{EPF}=14$

19 (P54) fig. 8

Forme: $\mathrm{O} /$ fond et bas de la panse.

Pâte : argileuse, orange vif, assez fine.

Trt. de surf. int. : engobe blanchâtre ; noeud de Salomon incisé. Glaçure caramel.

Trt. de surf. ext. : coulures de glaçure.

$\mathrm{DSP}=92 \mathrm{DIP}=88 \mathrm{IIIP}=15 \mathrm{HIP}=8 \mathrm{EPF}=10$

20 (IZ.95.65) fig. 8

Forme : $\mathrm{O} /$ fond.

Pâte : argileuse, orange clair, bien épurcé mais grossièrement travaillće.

Trt. de surf. int. : cngobe rosé ; au fond, sorte de fleur stylisée assez grossièrement, rehausséc de pigments bruns. Glaçure vert clair, brillante.

Traces de pernette : deux pieds arrachés et un arrachement de glaçure.

Trt. de surf. ext. : aucun.

$\mathrm{DSP}=80 \mathrm{DIP}=86 \mathrm{HEP}=12 \mathrm{HIP}=6 \mathrm{EPF}=14$

\section{1 (IZ.95.52) fig. 4, 7}

Forme : $O$ / bord et haut de la panse.

Pâte : argilcusc, orange, fine.

Trt. de surf. int. : engobe blanchâtre; sur lc haut du bord un bandeau de pigments vert sapin; puis dans un bandeau délimité par deux cercles concentriques, une ligne ondulée ; enfin sur le haut de la panse, un bandeau orné de chevrons alternés avcc unc sorte d'ecu hachuré. Glaçure jaune clair très brillante avec rehauts de pigments colorés verts et bruns plus ou moins en relation avec le motif incise.

Trt. de surf. ext. : engobe et glaçure sur le haut du bord.

DSUP $=240 \mathrm{HT}=80$

22 (IL.95.48) fig. 4, 7

Forme : $\mathrm{O} /$ bord à marli ct haut de pansc.

Pâte : argileuse, orange, fine, bien cuite.
Trt. de surf. int. : engobe blanchâtre ; sur te bord, deux cercles concentriques incisés puis bandeau de chevrons ; deux cercles concentriques après le point d'inflexion, puis trois cercles en haut de la panse et chevrons sur la panse. Glaçure jaune pâle très brillante et rehauts de pigments caramel et verts. Trt. de surf. ext. : engobe et glaçure sur lc bord, puis engobe seul.

DSUP $=200 \mathrm{DNL}=197 \mathrm{DPI}=154 \mathrm{DPIS}=137 \quad \mathrm{HT}=37$ $\mathrm{IINL}=30 \mathrm{HPI}=19 \mathrm{HPIS}=5$

23 (IZ.95.49) fig. 4

Forme : $\mathrm{O} /$ bord avec marli.

Pâte : argileuse, orange, fine, bien cuitc.

Trt. de surf. int. : engobe beige; deux cercles concentriques incisés sur le bord, puis trois cercles concentriques sur le point d'inflexion, sur la panse des demi-cercles. Glaçure vert pâle avec rehauts verts plus soutenus sur certains traits.

Trt. de surf. ext. : cngobe et glaçure vert clair sur le bord. DSUP $=210 \mathrm{DPI}=200 \mathrm{HT}=34 \mathrm{HPI}=21$

24 (IZ.95.50) fig. 4

Forme : $0 /$ bord.

Pâte : argileuse, orange clair, fine.

Trt. de surf. int. : engobe blanchâtre ; deux cercles concentriques incises sur le bord, puis bandeau de chevrons grossièrement incisés et lignes diverses sur la panse. Glaçure caramel. Trt. de surf. ext. : engobe et glaçure sur le haut du bord, puis engobe sur lc haut de la panse.

DSUP $=250 \mathrm{DPI}=240 \mathrm{HT}=29 \mathrm{HPI}=12$

25 (IZ.95.51) fig. 4

Forme : $0 /$ bord.

Pâte : argilcuse, orange clair, fine, bien cuite.

Trt. de surf. int. : engobc rosé ; deux cercles concentriques incisés sur le bord, puis un bandeau de chevrons et un cercle concentrique. Glaçure jaune brillante avec rehauts de pigments bruns et vert foncé.

Trt. de surf. ext. : engobe et glaçure verte sur le haut du bord, puis engobe seul.

$\mathrm{DSUP}=260 \mathrm{DPI}=248 \mathrm{HT}=29 \mathrm{HPI}=22$

\section{Vases rejetés en cours d'élaboration}

26 (P1) fig. 8

Forme : $\mathrm{O} /$ fond.

Pâte : argileuse, orange, fine.

Trt. de surf. int. : engobe rosé ; dans un large médaillon délimité par deux cercles concentriques incisés, une fleur à cinq pétales, les espaces entre les pétales sont hachurés.

Trt. de surf. ext. : aucun.

$\mathrm{DSP}=63 \mathrm{DIP}=70 \mathrm{HEP}=12 \mathrm{HIP}=12 \mathrm{LPI}=10$

\section{7 (IZ.95.66) fig. 5}

Forme : $\mathrm{O} /$ fond.

Pâte : argileuse, ocre, fine, bien épurée.

Trt. de surf. int. : cngobe beige ; dans un large médaillon central délimité par deux cercles concentriques, une fleur à huit 
pétales incisée. Une tache de glaçure verte.

Trt. de surf. ext. : aucun. Sous l'anneau de la base, trois marques de pernette collée, autour de ces marques glaçure vert foncé, comme si les pieds de la pernette collée, tachés, avaient coulé pendant la cuisson. Sous la base, un cône.

$\mathrm{DSP}=78 \mathrm{DIP}=84 \mathrm{HEP}=11 \mathrm{HIP}=9 \mathrm{EPF}=12$

\section{8 (IZ.95.6.3)}

Forme : $\mathrm{O} /$ fond.

Pâte : argilcuse, orange, fine mais grossic̀rement travailléc.

Trt. de surf. int. : aucune trace d'engobe ; dans un large médaillon occupant tout le fond, délimité par un cercle concentrique, une fleur incisée. Glaçure vert foncé, brillante.

Traces de pernette : deux arrachements énormes.

Trt. de surf. ext. : aucun. Sous l'anneau de la base, un morceau de pernette est resté collé.

$\mathrm{DSP}=74 \mathrm{DIP}=80 \mathrm{HEP}=9$

29 (IZ.95.50 bis) fig. 2,6

Forme : $\mathrm{O} /$ fond.

Pâte : argileuse, ocre, bien épurée mais grossièrement travaillée.

Trt. de surf. int. : engobe beige ; lignes rayonnantes incisées. Trt. de surf. ext. : aucun.

$\mathrm{DSP}=80 \mathrm{DIP}=84 \mathrm{HEP}=13 \mathrm{HIP}=7 \mathrm{FPF}=11$

30 (IZ.95.68) fig. 3, 6

Forme : $\mathrm{O} /$ fond et bas de panse.

Pâte : argileuse, ocre, fine.
Trt. de surf. int. : engobe beige ; médaillon central orné de hachures - dans le genre des blasons mamelouks - puis série de quatre cercles concentriques incisés, sur la panse, spirales incisées.

Trt. de surf. ext. : aucun. Traces de tournage trc̀s nettes.

$\mathrm{DSP}=85 \mathrm{DIP}=88 \mathrm{HLP}=8 \mathrm{HIP}=10 \mathrm{EPF}=10$

31 (IZ.95.69) fig. 3, 6

Forme: $O /$ fond et bas de la panse.

Pâte : argileuse, ocre, fine, bien épurćc.

Trt. de surf. int. : engobe beige ; dans un médaillon central, succession de chevrons couronnée d'un zig-zag, puis śur le bas de la panse, quatre cercles concentriques et un zig-zag.

Trt. de surf. ext. : aucun.

$\mathrm{DSP}=83 \mathrm{DIP}=86 \mathrm{HEP}=14 \mathrm{HIP}=16 \mathrm{EPF}=9$

32 (IZ.95.21) fig. 4, 7

Forme : $\mathrm{O} /$ bord et haut de la panse.

Pâte : argileuse, orange clair, assez fine avec petites inclusions blanches.

Trt. de surf. int. : engobe beige ; trois cercles concentriques incisés sur le bord, puis une ligne ondulee, deux cercles concentriques et une ligne ondulćc.

Trt. de surf. ext. : engobe sur tout le fragment.

DSUP $=220 \mathrm{HT}=52$

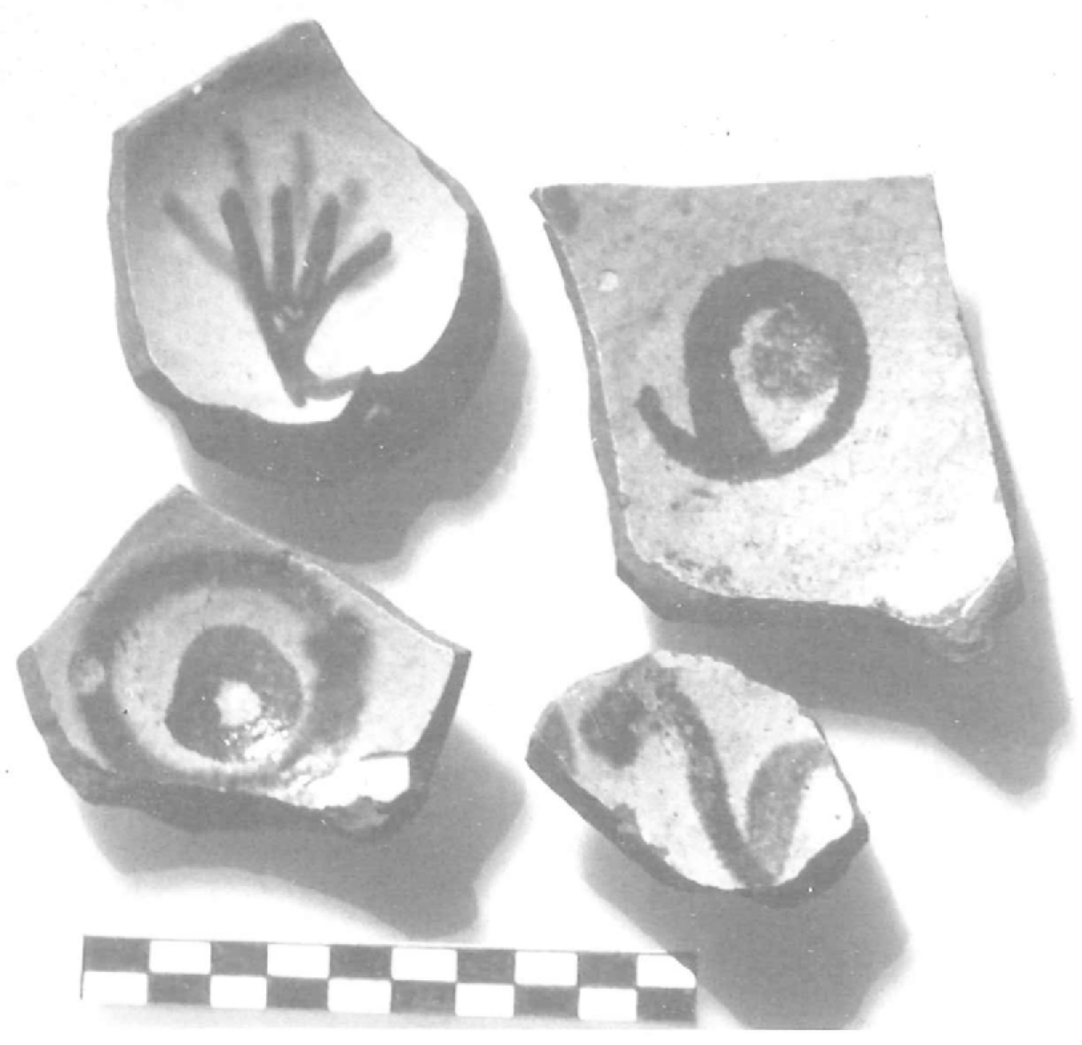



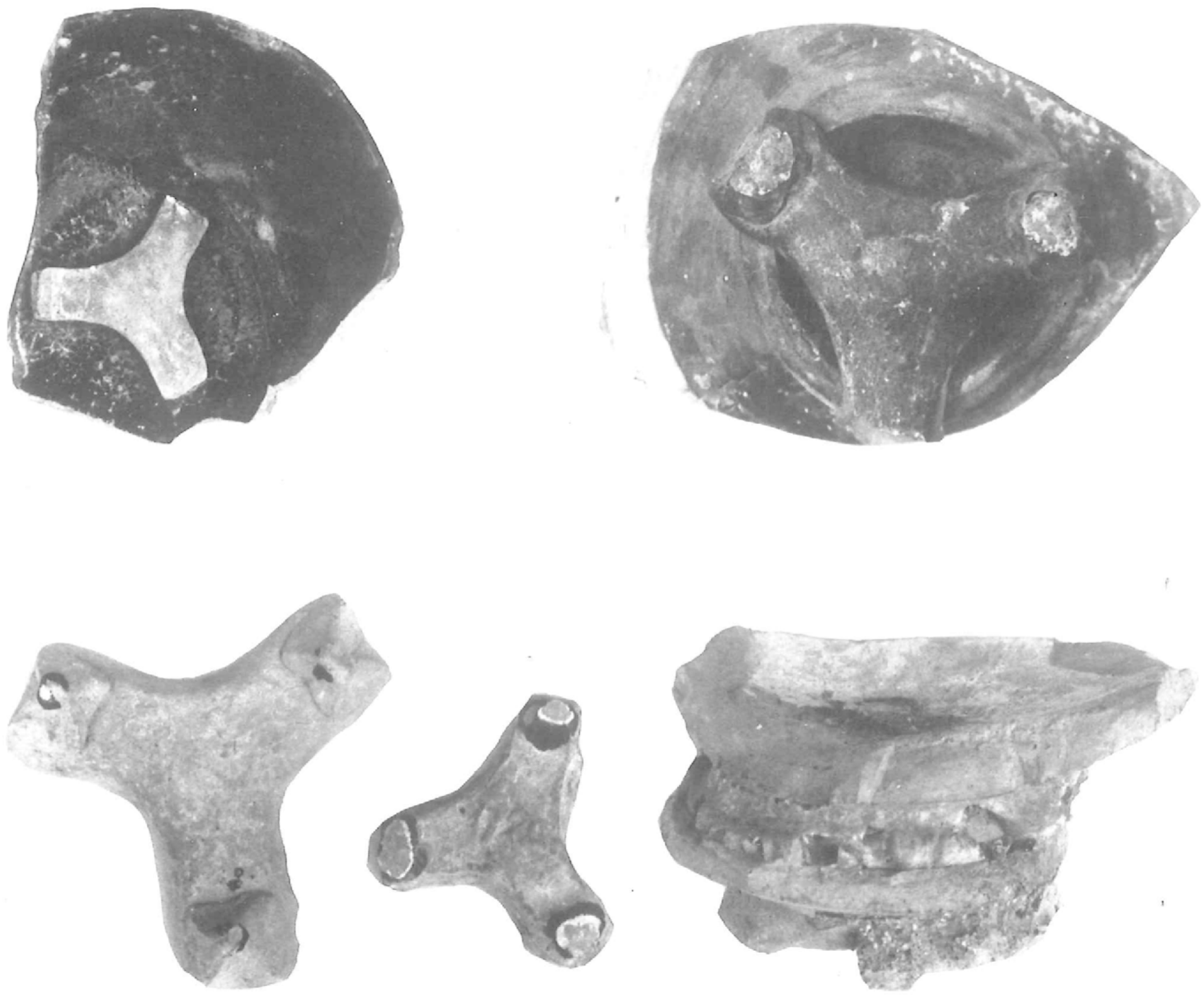

Fig. 1 : Ratés de cuisson et pernettes. 


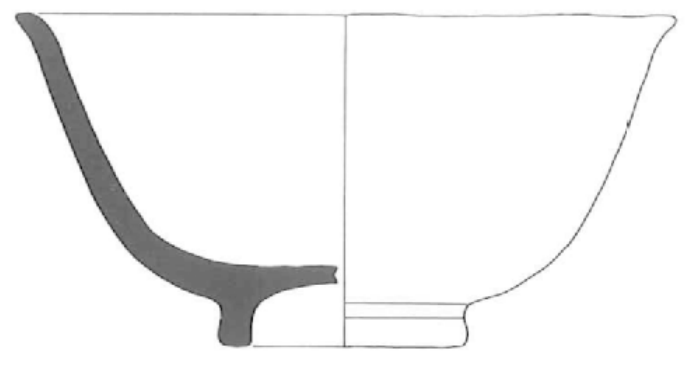

10
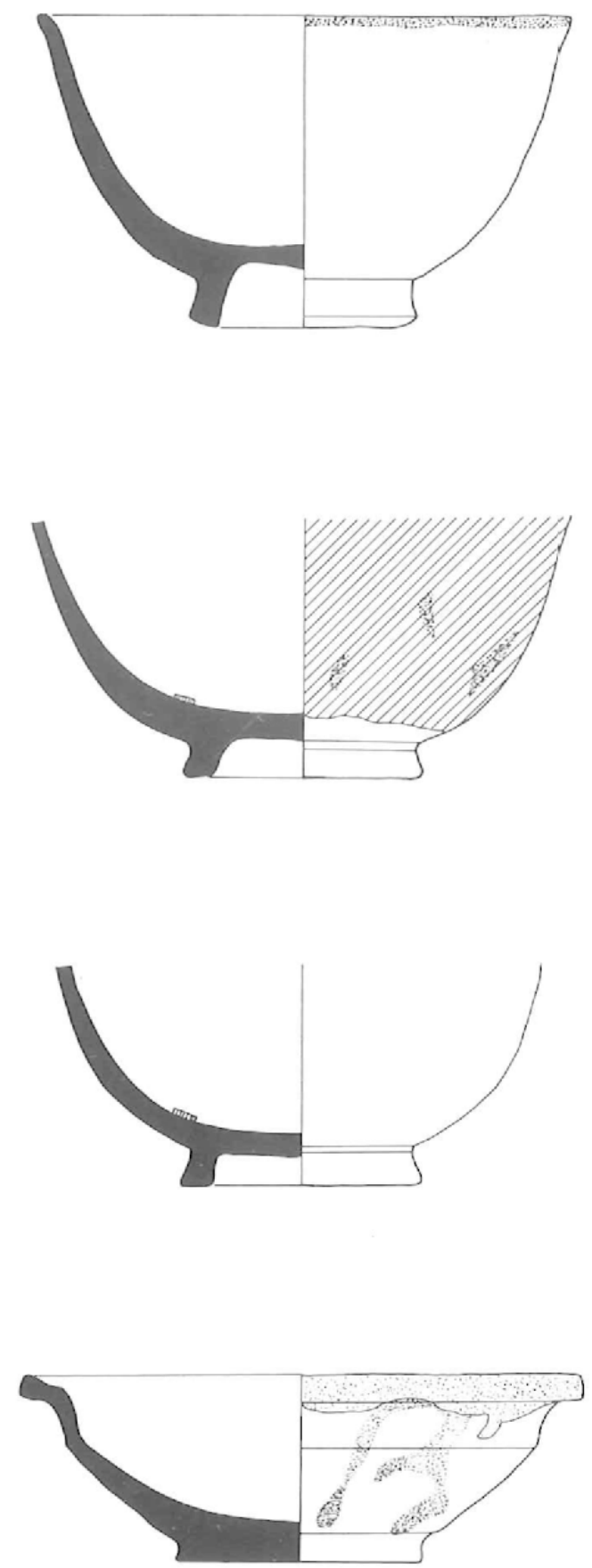
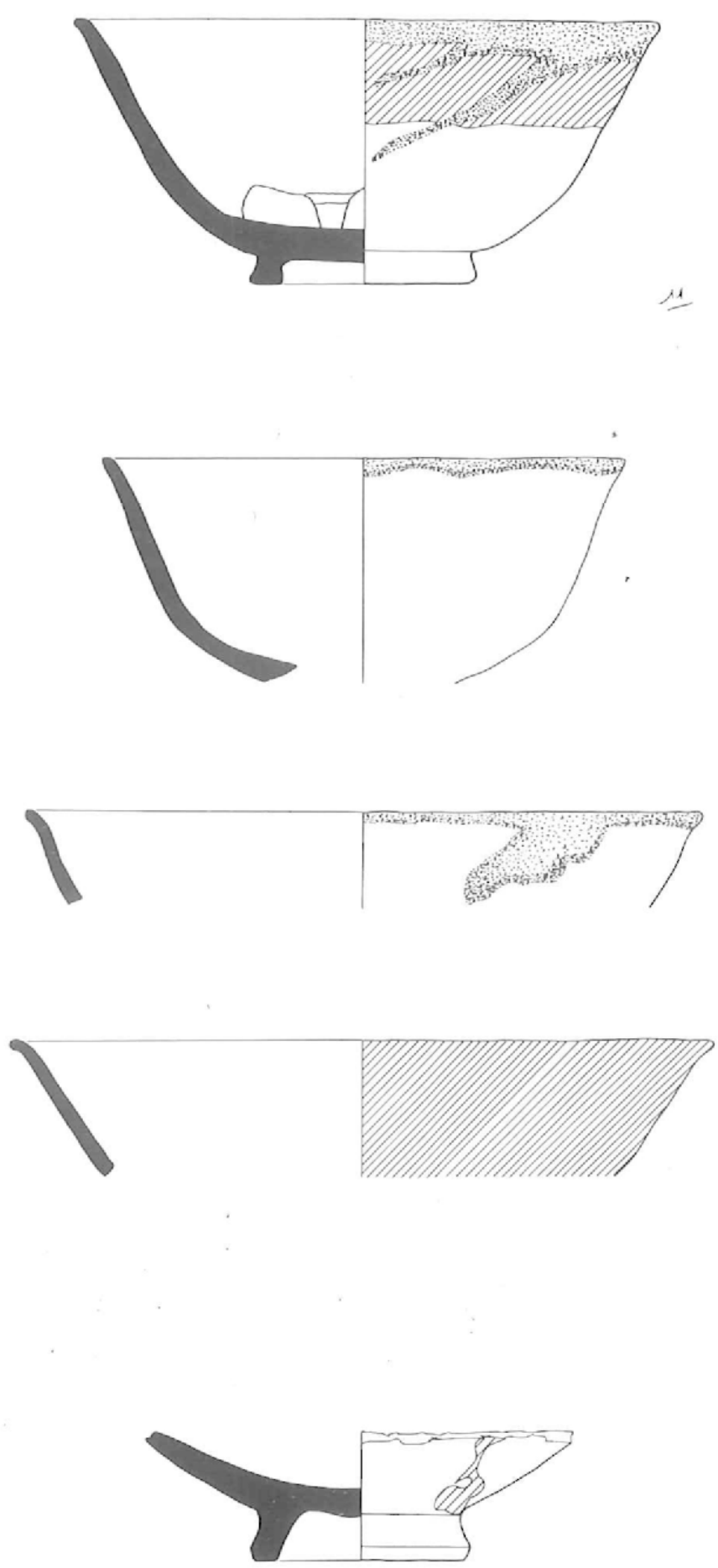

但

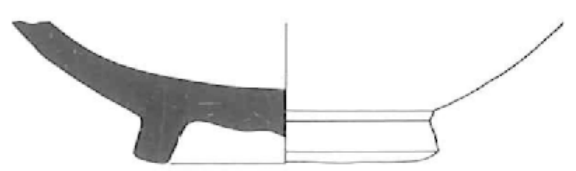

Fig. 2 

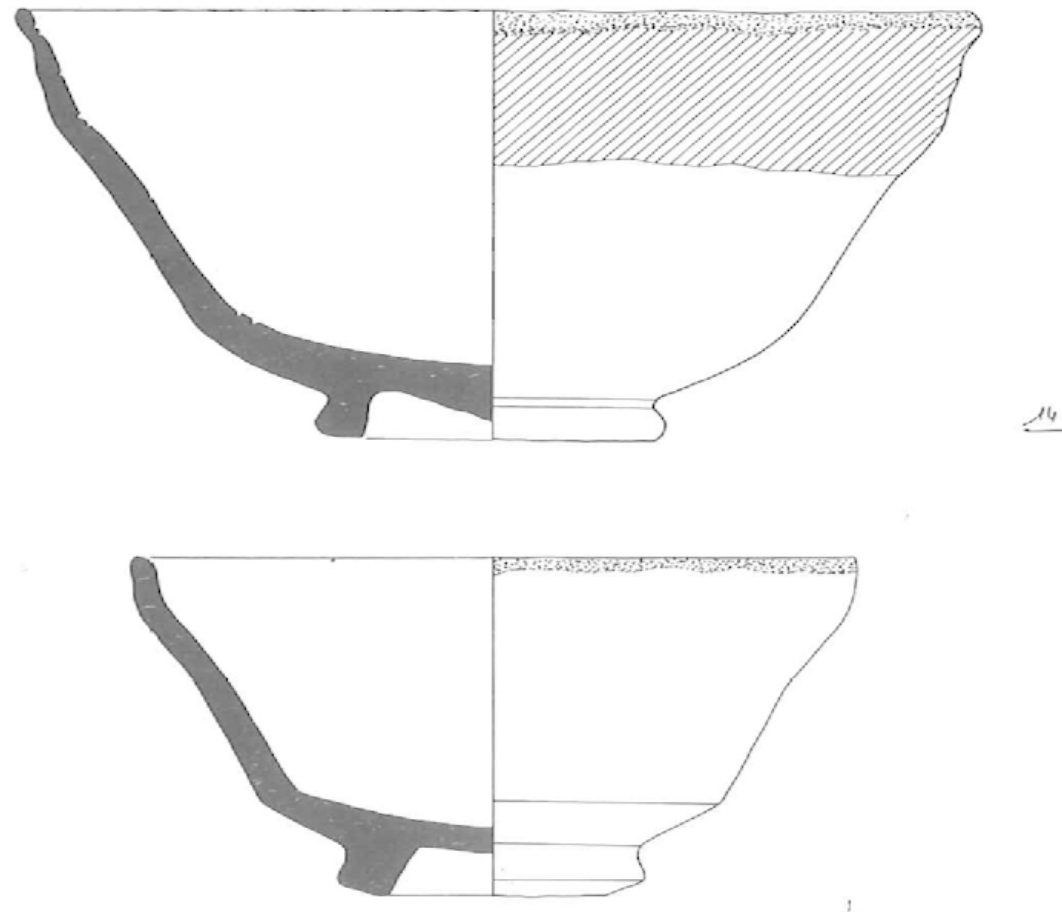

$\underline{2}$
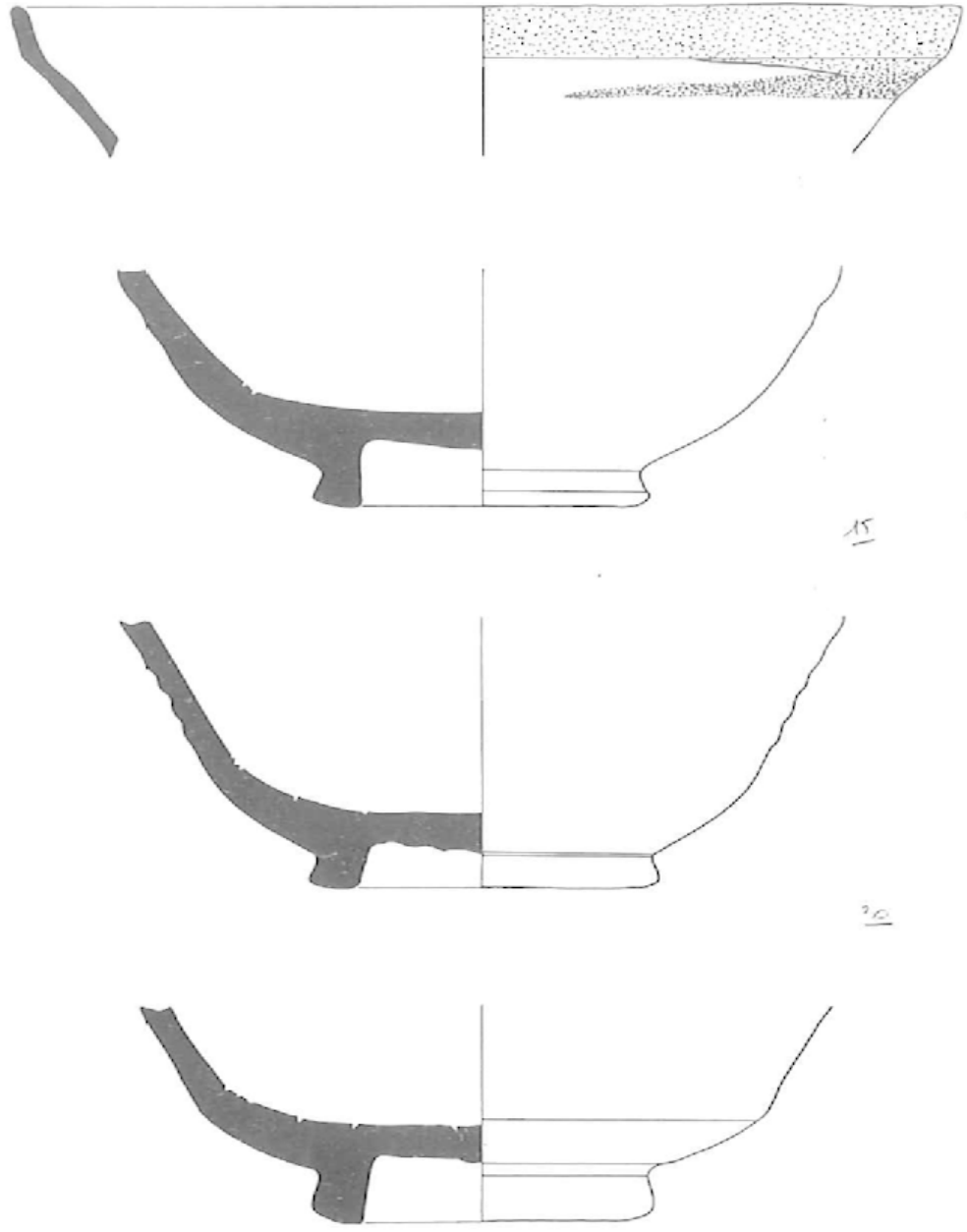

Fig. 3 


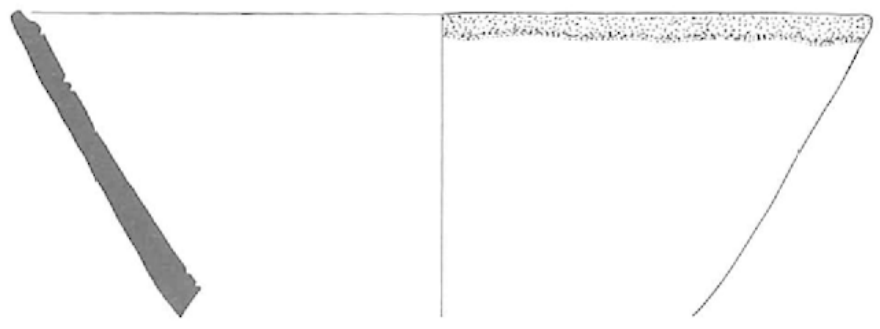

21

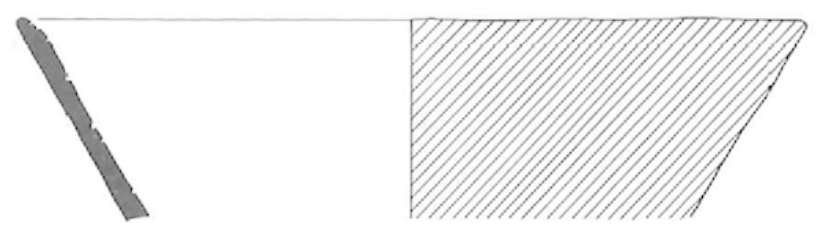

32.
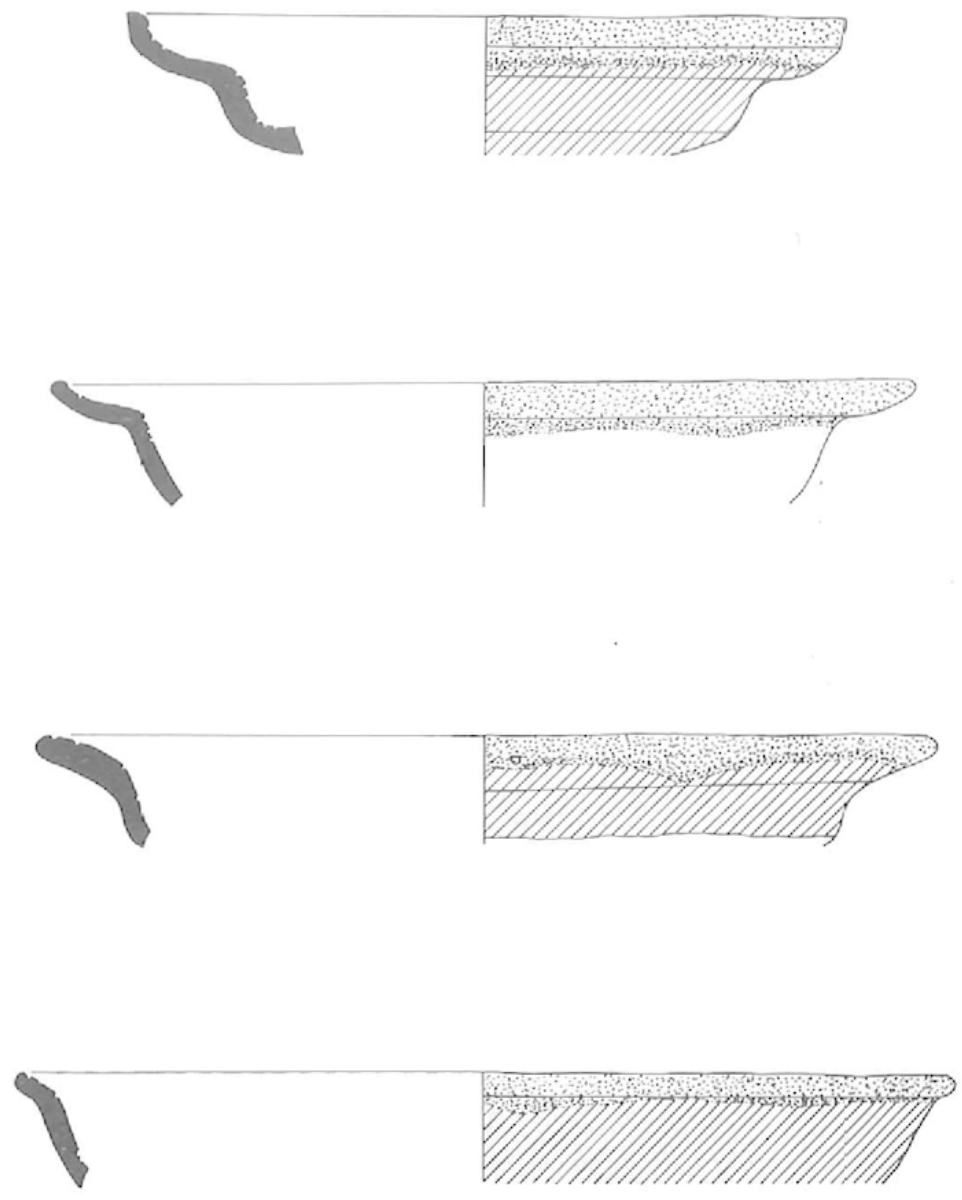

Fig. 4 

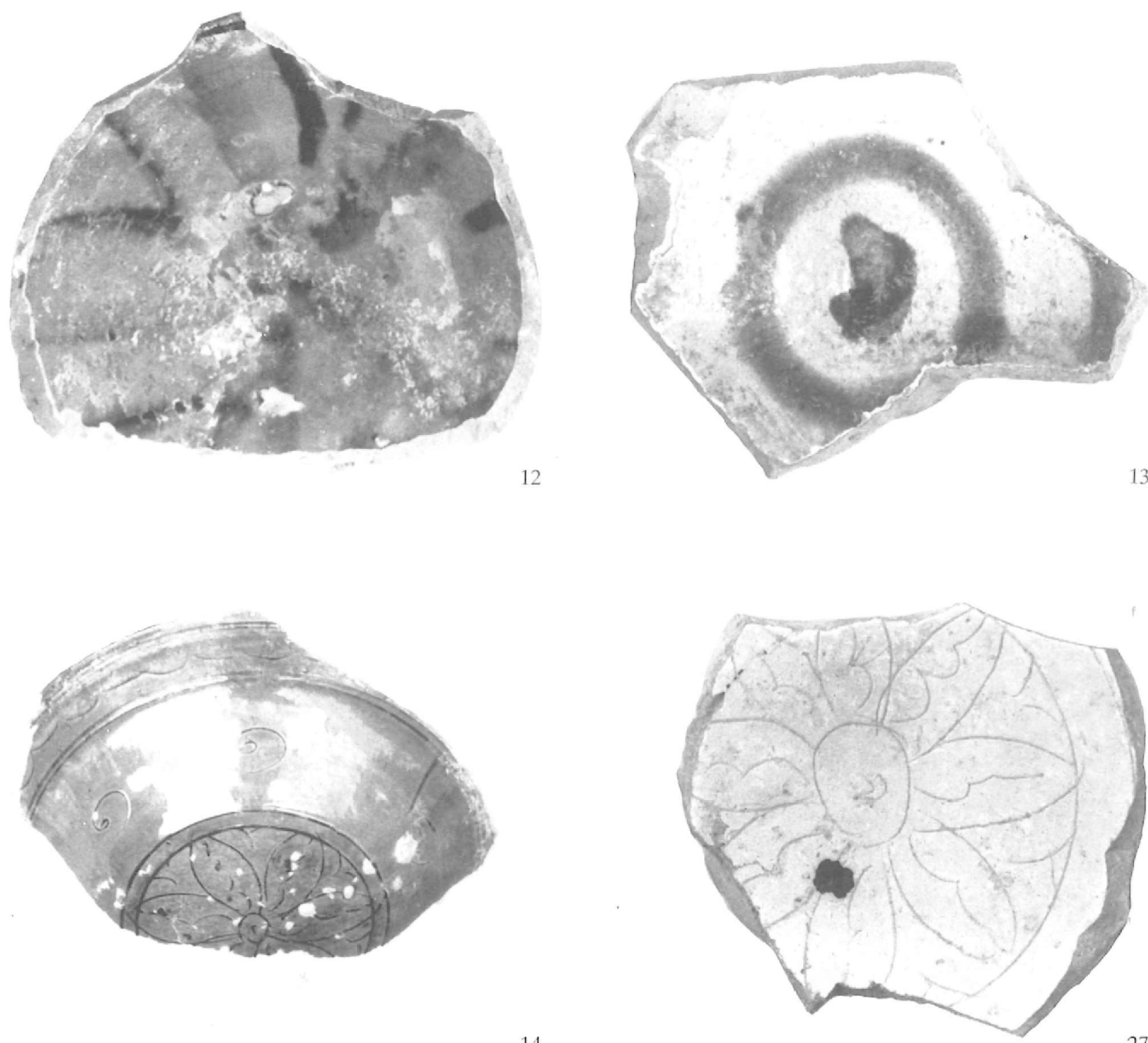

Fig. $5: 12,13$, eéramique-pointe-de-Fhasos, 14, 27. 

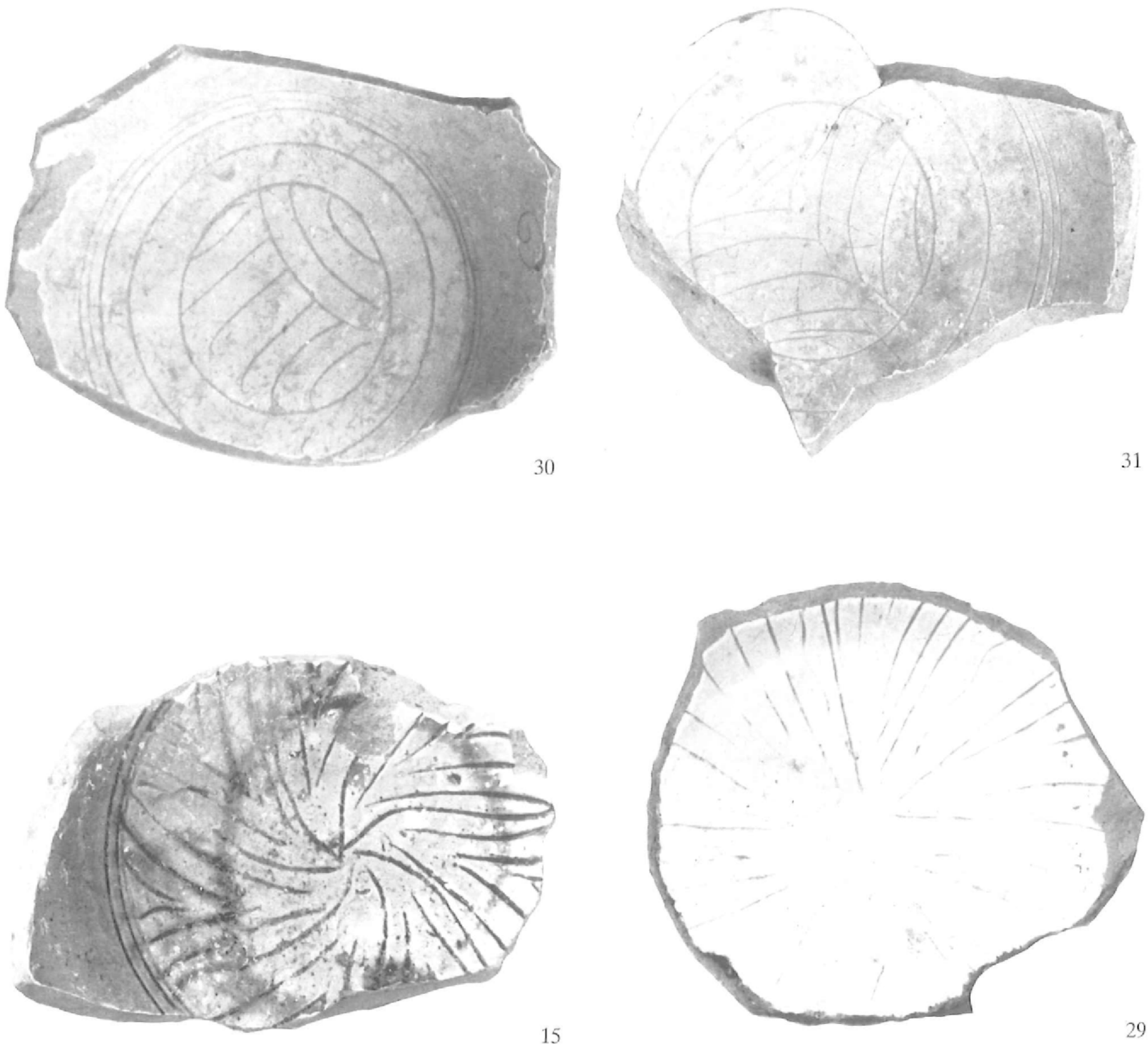

Fig. $6: 30,31,15,29$. 

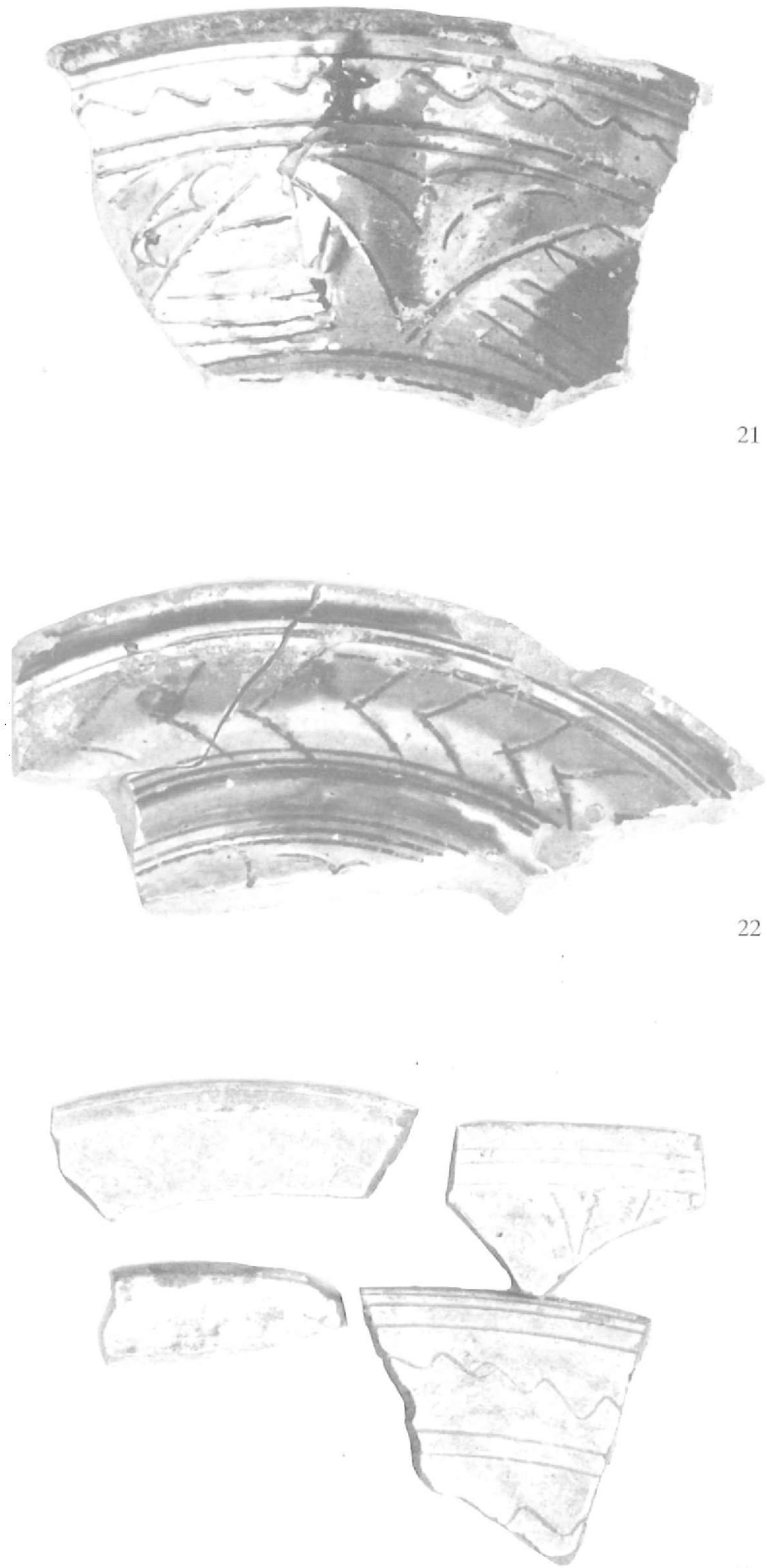

Fig. 7 : 21, 22, 32. 

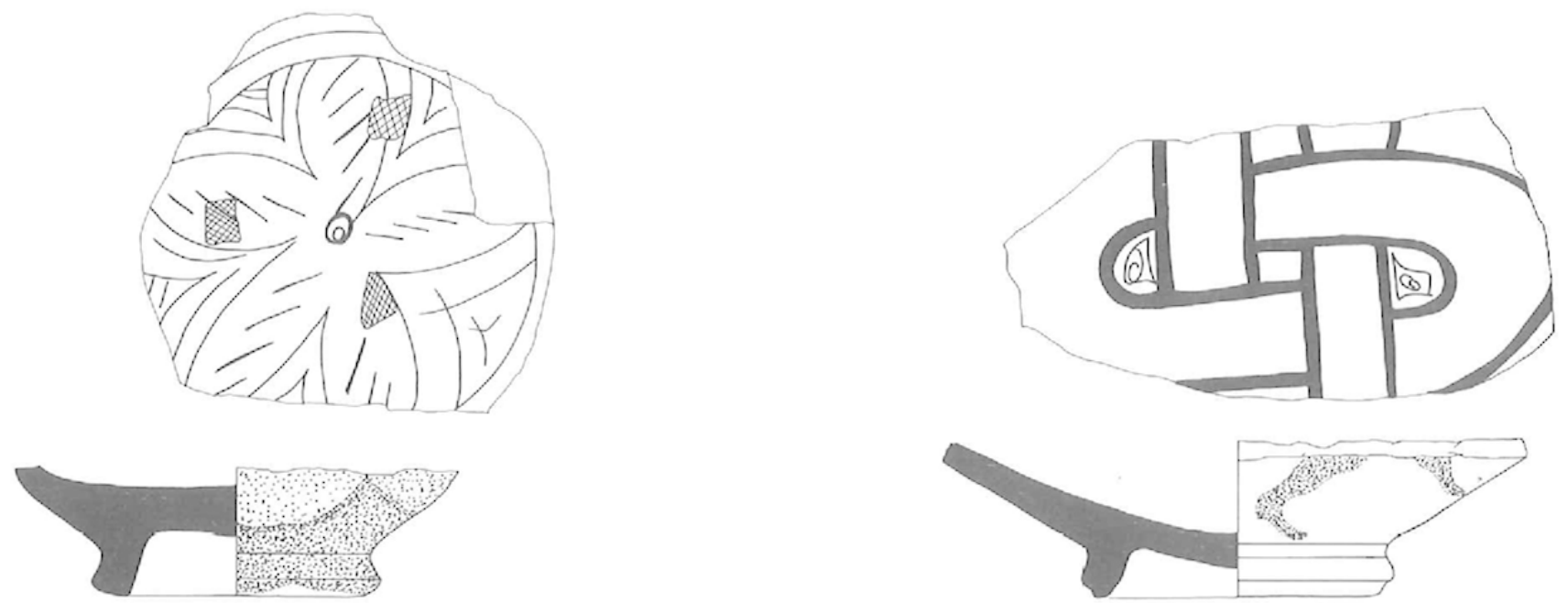

19
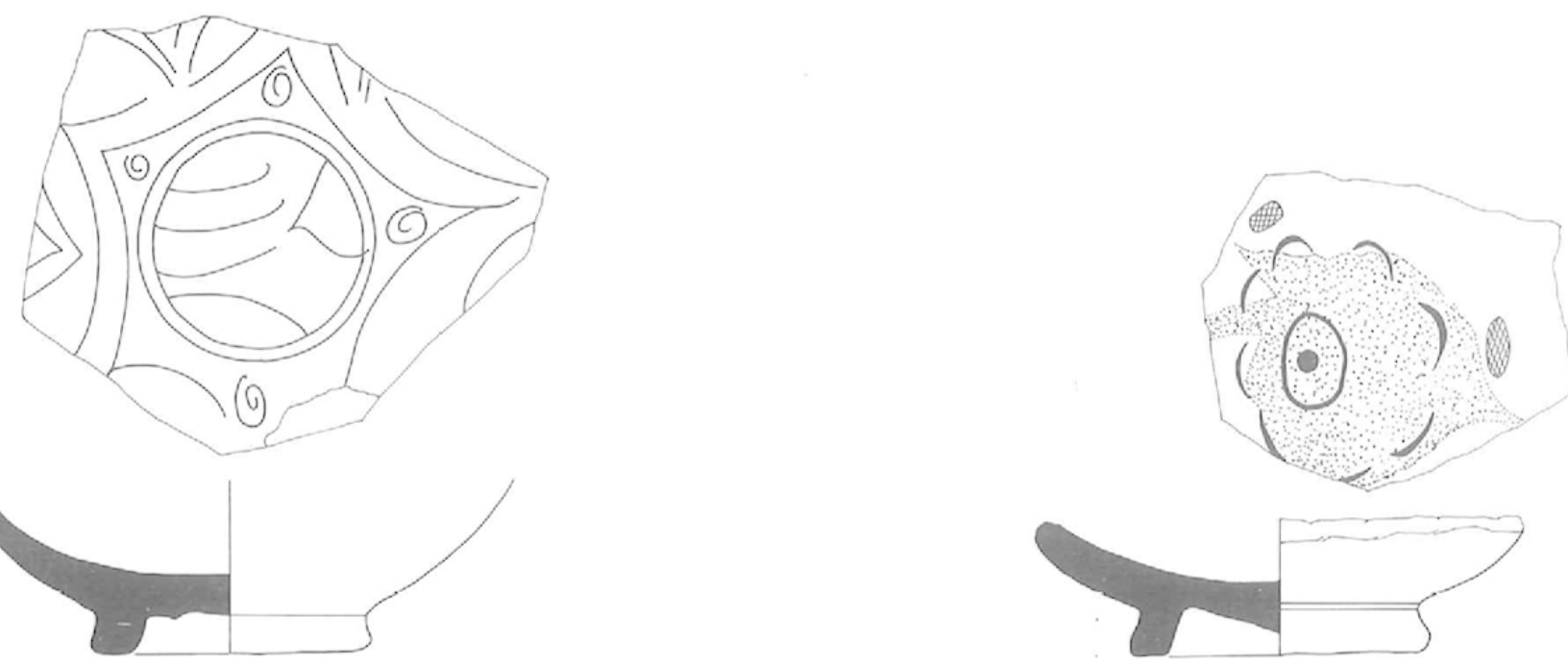

17
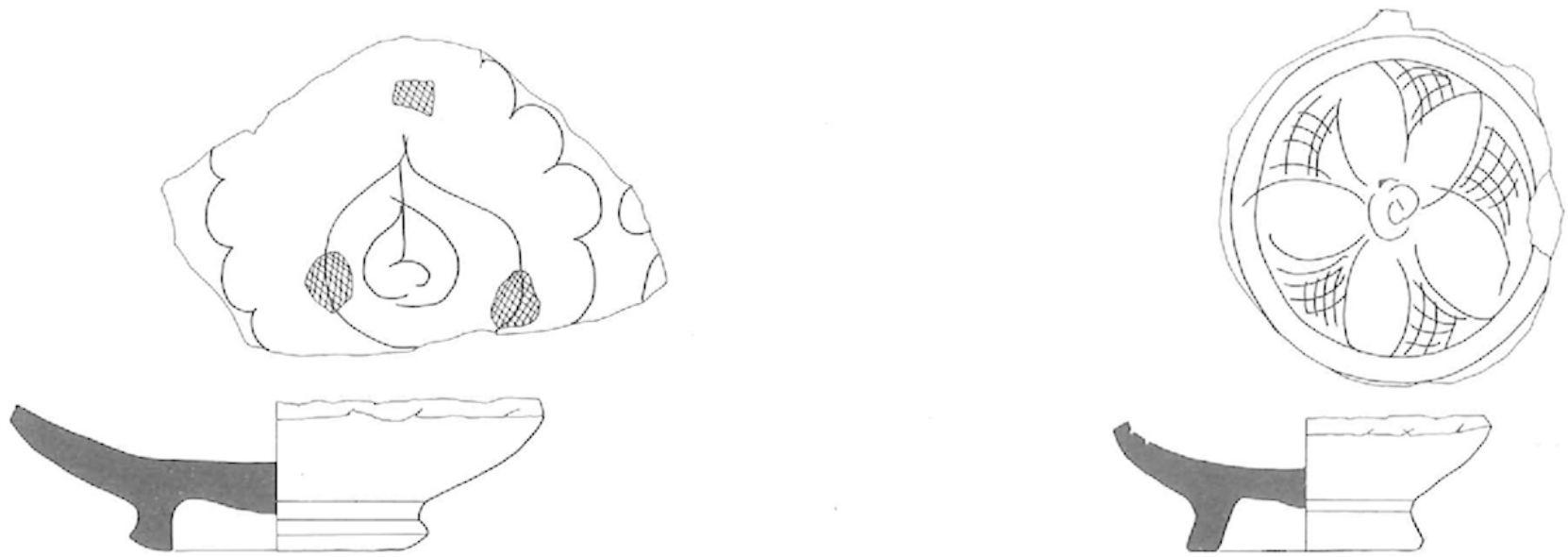

Fig. 8 